\title{
Article
}

\section{Generalizations of the R-Matrix Method to the Treatment of the Interaction of Short Pulse Electromagnetic Radiation with Atoms}

\author{
Barry I. Schneider ${ }^{1}$ 0000-0001-8510-499X, Kathryn R. Hamilton ${ }^{2}{ }^{-0000-0002-8245-0122, ~ a n d ~ K l a u s ~}$ \\ Bartschat $^{2}$ (D)000-0001-6215-5014 \\ 1 Applied and Computational Mathematics Division, National Institute of Standards and Technology, \\ Gaithersburg, MD 20899; bis@nist.gov \\ 2 Department of Physics and Astronomy, Drake University, Des Moines, IA 50311, USA; \\ kathryn.hamilton@drake.edu; klaus.bartschat@drake.edu \\ * Correspondence: bis@nist.gov
}

\begin{abstract}
Since its initial development in the 1970's by Phil Burke and his collaborators, the R-matrix theory and associated computer codes have become the de facto approach for the calculation of accurate data for general electron-atom/ion/molecule collision and photoionization processes. The use of a non-orthonormal set of orbitals based on B-splines, now called the B-spline R-matrix (BSR) approach, was pioneered by Zatsarinny. It has considerably extended the flexibility of the approach and improved particularly the treatment of complex many-electron atomic and ionic targets, for which accurate data are needed in many modelling applications for processes involving low-temperature plasmas. Both the original R-matrix approach and the BSR method have been extended to the interaction of short, intense electromagnetic (EM) radiation with atoms and molecules. Here we provide an overview of the theoretical tools that were required to facilitate the extension of the theory to the time domain. As an example of a practical application, we show results for two-photon ionization of argon by intense short-pulse extreme ultraviolet radiation.
\end{abstract}

Keywords: B-spline R-matrix, R-matrix with time dependence, intense short-pulse extreme ultraviolet radiation, time-dependent Schrödinger equation; Arnoldi-Lanczos propagation

\section{Introduction}

The evolution of R-matrix theory from a useful but largely phenomenological approach for the description of nuclear resonances $[1,2]$ to an accurate computational method in atomic physics in the 1970's is primarily due to Phil Burke and his collaborators at The Queen's University of Belfast [3-6]. The refinement of the original theory to include long-range interactions and the associated computer codes have become the de facto approach for the calculation of accurate data for general electronatom/ion/molecule collision and photoionization processes in general and cross sections in particular. These data are needed for many modeling applications for processes involving low-temperature plasmas, in addition to providing critical insights into how electrons and photons interact with atomic systems. The atomic R-matrix methods were subsequently extended to the study of electron-molecule collisions [7-9] and ultimately led to the now widely used UKRmol+ package [10].

A significant advance in the practical use of the R-matrix method was made by Oleg Zatsarinny when he introduced a non-orthogonal (NO) basis set representation of the wavefunction using B-splines. The B-spline R-matrix (BSR) approach [11,12] is based on an idea that had been very successfully introduced into atomic structure calculations by Charlotte Froese Fischer [13].

Oleg pioneered using non-orthogonal sets of orbitals in the R-matrix method and showed that by using a flexible radial B-spline basis, it was possible to greatly improve 
the accuracy of the calculated results. The NO basis enables the efficient incorporation of correlation into the atomic expansion, using individually optimized, i.e., term-dependent one-electron orbitals. The B-splines provide a very accurate description of the atomic bound and continuum orbitals, together with excellent and well-studied numerical properties. B-splines as the primitive numerical basis, together with their use to expand the non-orthogonal set of physical and pseudo-orbitals in them, provide a potent mix that may improve significantly on the standard versions of the atomic R-matrix codes.

Recently, the finite-difference representation of the orbitals in the well-known atomic code [14] has indeed been replaced by B-splines [15]. At this point, however, the individual orbitals expanded in this basis are still assumed to be orthogonal, thereby limiting their flexibility to represent the generally existing term dependence. In principle, of course, this term dependence can be represented in large configuration-interaction expansions, as done very successfully in the convergent close-coupling (CCC) [16] and R-matrix with pseudo-states (RMPS) [17] formulations. Hence, while nonorthogonal orbital sets are not an advantage per se in the general formulation of close-coupling theory, they can be highly beneficial in reducing the required size of the expansion in practical applications. Undoubtedly, they come with their own challenges, which is the main reason they tend not to be used in collision problems, where both the $N$-electron target and the $(N+1)$-electron collision problem need to be handled at a comparable and consistent level of sophistication. A significant difference between atomic and molecular structure calculations and those involving scattering is that the former more commonly employ methodologies that optimize orbitals for specific states. In studying dynamical systems where there is a strong interplay between neutral, excited and ionic states, this is far greater challenge.

The BSR method has been demonstrated to be capable of computing highly accurate electron-collision and photoionization amplitudes and cross sections for complex atomic and ionic targets. There are now non-relativistic, semi-relativistic (Breit-Pauli), and full-relativistic (Dirac-Coulomb) versions. In some cases, the BSR calculations were shown to be more accurate than the available experimental data, and sometimes the calculations suggested strong evidence of problems with existing experimental data that were later corrected by newer experiments. For just one such example, see Ren $e t$ al. [18].

Around 2006-2007, it became clear that input from BSR calculations could be used to treat the interaction of intense laser radiation with complex atoms such as $\mathrm{Ne}$ [19] and Ar [20]. All the key ingredients were available; namely, the Hamiltonian matrix and the dipole length and/or velocity matrix elements required for the coupling of the target system to the external EM field. Since these could be "easily" extracted from the BSR code, this would provide a general ab initio and non-perturbative treatment to the problem that goes beyond quasi-one-electron or quasi-two-electron models.

The only other required ingredient is an efficient method to propagate the timedependent wavefunction and a way to extract the information from that wavefunction at long times, when i) the external field has died down and ii) a possibly ejected electron has moved sufficiently far away from the target center. Schneider, Bartschat, and Guan had been working on the numerical propagation techniques using the short iterative Lanczos (SIL) method, and they convinced Oleg that using his BSR matrix elements would be a very useful contribution to atomic physics. The four of us combined our talents to examine the multi-photon, single-ionization of $\mathrm{Ne}$ and $\mathrm{Ar}$, which are significantly more challenging targets than $\mathrm{He}$ for this process. Eventually, the method that we hereafter term TDBSR, was generalized to treat two-photon, double ionization of He [21,22]. The late Cliff Noble also joined the group and was instrumental in parallelizing the codes we had developed. With the addition of Johannes Feist, we later examined the question of time delays in the photoionization of $\mathrm{Ne}$ [23].

The generalization of the Belfast R-matrix method to treat arbitrary time-dependent EM fields actually goes much further back to a paper written by Phil and Valerie Burke in 1997 [24]. Subsequent theoretical [25] developments lead to the generation of the 
Time-Dependent R-matrix method (TDRM) [26], which used a B-spline basis approach in the R-matrix inner region. Combining this with an outer region based on the finite difference techniques used in the HELIUM code [27] led to the first incarnation of the R-matrix with time dependence method (RMT) [28] in 2011. Since then the code has undergone extensive redevelopment, with extensions to treat arbitrary light fields [29], molecular targets [30], and spin-orbit interaction using input from the RMATRX-I suite of codes [31], and improvements to the parallelization scheme [32]. It is still under active development by researchers located mostly in the UK.

Both the BSR code and the RMT suite are available from a number of sources, such as Computer Physics Communications [11,32] and designated github sites [33,34]. A good place to start with these and other codes in this field is the Atomic, Molecular, and Optical Sciences (AMOS) Gateway [35].

In this contribution, we take the opportunity to examine and contrast how the TDBSR and RMT methods are structured today. Specifically, we highlight the contributions of the collaboration between Schneider at NIST and the Bartschat group at Drake University to this topic. The basic ideas are outlined in Section 2. As one representative example, we compare the TDBSR and RMT results for two-photon ionization of Ar atoms by an intense short-pulse extreme ultraviolet (XUV) laser field in Section 3. We finish in Section 4 with a summary and an outlook to suggest that a very worthwhile long-term goal is the unification or at least the interoperability of the two codes through the development of an appropriate interface.

\section{Solving the TDSE via the R-Matrix Method}

The general solution of the time-dependent Schrödinger equation (TDSE) in the internal R-matrix region is

$$
\begin{aligned}
& {\left[i \frac{\partial}{\partial t}-\mathcal{K}\left(\mathbf{X}_{N+1}\right)-\mathcal{D}\left(\mathbf{X}_{N+1}, t\right)\right]\left|\Psi\left(\mathbf{X}_{N+1}, t\right)\right\rangle=-\mathcal{L}\left|\Psi\left(\mathbf{X}_{N+1}, t\right)\right\rangle} \\
& \mathcal{K}\left(\mathbf{X}_{N+1}\right)=\mathcal{H}\left(\mathbf{X}_{N+1}\right)+\mathcal{L} \\
& \mathcal{L}=\sum_{\alpha} L_{\alpha} \\
& L_{\alpha}=\frac{1}{2} \sum_{i}\left|\phi_{\alpha}\left(\mathbf{X}_{N}\right)\right\rangle \delta\left(r_{i}-R_{0}\right)\left[\frac{\partial}{\partial r_{i}}-b\right]\left\langle\phi_{\alpha}\left(\mathbf{X}_{N}^{\prime}\right)\right| .
\end{aligned}
$$

where $\mathbf{X}_{q}=\left[\mathbf{x}_{1}, \mathbf{x}_{2}, \mathbf{x}_{3} \ldots, \mathbf{x}_{q}\right]$ and $\mathbf{x}_{i}=\mathbf{r}_{i} \sigma_{i}$ represents the space and spin coordinates of electron $i$. Here $\mathcal{K}\left(\mathbf{X}_{N+1}\right)$ is the time-independent Hamiltonian, $\mathcal{D}\left(\mathbf{X}_{N+1}, t\right)$ represents the interaction of the system with the EM field, $\mathcal{L}$ is the Bloch operator, and $\mathbf{X}_{N+1}$ is used to label the entire set of $(N+1)$ internal coordinates of the system. The states $\left|\phi_{c}\right\rangle$ that are being summed over in Eq. (1c) are the channel functions that are non-zero at the $\mathrm{R}$-matrix boundary $R_{0}$. Their definition depends on how asymptotically exact quantum states are constructed as the interaction between the target and projectile approaches zero. Once that choice has been made, those parts of the wavefunction involving the internal coordinates $\mathbf{X}_{N}$ of the target and the projectile can be coupled to form the channel functions, and those can be projected out of the calculation leaving only the 
wavefunction in the relative coordinate $r$ in the asymptotic region to be determined. In the external region, therefore, we have a time-dependent one-electron problem:

$$
\begin{gathered}
\\
{\left[i \frac{\partial}{\partial t}-\mathbf{h}(r)-\mathbf{d}(r, t)\right] \mathbf{f}(r, t)=\mathbf{L f}(r, t) ;} \\
\mathbf{h}(r)+\mathbf{d}(r, t)=\left(\begin{array}{ccccc}
h_{1,1}(r, t) & h_{1,2}(r, t) & h_{1,3}(r, t) & \cdots & h_{1, n}(r, t) \\
h_{2,1}(r, t) & h_{2,2(r, t)} & h_{2,3}(r, t) & \cdots & h_{2, n}(r, t) \\
h_{3,1}(r, t) & h_{3,2(r, t)} & h_{3,3}(r, t) & \cdots & h_{3, n}(r, t) \\
\vdots & \vdots & \vdots & \ddots & \vdots \\
h_{n, 1}(r, t) & h_{n, 2}(r, t) & h_{n, 3}(r, t) & \cdots & h_{n, n}(r, t)
\end{array}\right) ; \\
\mathbf{f}(r, t)=\left(\begin{array}{c}
f_{1}(r, t) \\
f_{2}(r, t) \\
f_{3}(r, t) \\
\vdots \\
f_{n}(r, t)
\end{array}\right) ; \\
h_{i, j}(r, t)=-\frac{1}{2} \frac{d^{2}}{d r^{2}}+\frac{l_{\alpha}\left(l_{\alpha}+1\right)}{2}+v_{\alpha, \beta}(r)+d_{\alpha, \beta}(r, t) .
\end{gathered}
$$

${ }_{111}$ The bold-faced notation represents matrices and vectors in the channel space. The explicit inclusion of the Bloch operator in both the internal and external region is done to emphasize that it is this term that couples the two regions. Note that it is entirely independent of the specific numerical method used to solve the set of equations in the two regions. In the current implementation of RMT [32], a five-point finite difference (FD) is used to represent the first and second derivatives.

Since the value of the logarithmic derivative to the solution to the TDSE in the inner and outer region must be the same at $R_{0}$,

$$
\begin{aligned}
{\left.\left[\frac{\partial}{\partial r}-b\right]\left(\psi_{\alpha} \mid \Psi\left(\mathbf{X}_{N+1}, t\right)\right)\right|_{r=R_{0}} } & =\left.\left[\frac{\partial}{\partial r}-b\right] f_{\alpha}(r, t)\right|_{r=R_{0}} ; \\
\left.\left(\psi_{\alpha} \mid \Psi\left(\mathbf{X}_{N+1}, t\right)\right)\right|_{r=R_{0}} & =\left.f_{\alpha}(r, t)\right|_{r=R_{0}} .
\end{aligned}
$$

The round brackets mean integration over all degrees of freedom save $r$. In a somewhat unorthodox fashion, one can replace the operation of $\mathcal{L}\left|\Psi\left(\mathbf{X}_{N+1}, t\right)\right\rangle$ on the right-hand side of Eq. (1a) with the values of the projected external wavefunction and, similarly, $\mathbf{L} \mathbf{f}(r, t)$ on the right-hand side of Eq. (2a) with the projected $\left|\Psi\left(\mathbf{X}_{N+1}, t\right)\right\rangle$. Then there are additional coupling terms due to approximating the derivatives of $f_{\alpha}(r, t)$ by difference formula. At present, the formalism only treats cases where a single free electron can be explicitly in the external region. This is a restriction of the current R-matrix formalism. Removing it is a fundamental challenge. Equations (1a) and (2a) can be elegantly rewritten using the identity,

$$
\begin{aligned}
i \frac{\partial}{\partial t}\left[\exp \left(i \mathcal{K}\left(\mathbf{X}_{N+1}\right) t\right)\left|\Psi\left(\mathbf{X}_{N+1}, t\right)\right\rangle\right] & = \\
& -\mathcal{K}\left(\mathbf{X}_{N+1}\right) \exp \left(i \mathcal{K}\left(\mathbf{X}_{N+1}\right) t\right)\left|\Psi\left(\mathbf{X}_{N+1}, t\right)\right\rangle \\
& +\exp \left(i \mathcal{K}\left(\mathbf{X}_{N+1}\right) t\right) i \frac{\partial}{\partial t}\left|\Psi\left(\mathbf{X}_{N+1}, t\right)\right\rangle
\end{aligned}
$$

and then integrating between $t_{0}$ and $t$ to obtain

$$
\begin{gathered}
\left|\Psi\left(\mathbf{X}_{N+1}, t\right)\right\rangle=\exp \left(-i \mathcal{K}\left(\mathbf{X}_{N+1}\right)\left(t-t_{0}\right)\right)\left|\Psi\left(\mathbf{X}_{N+1}, t_{0}\right)\right\rangle \\
-i \exp \left(-i \mathcal{K}\left(\mathbf{X}_{N+1}\right) t\right) \int_{t_{0}}{ }^{t} d t^{\prime} \exp \left(i \mathcal{K}\left(\mathbf{X}_{N+1}\right) t^{\prime}\right)\left[\mathcal{D}\left(\mathbf{X}_{N+1}, t^{\prime}\right)-\mathcal{L}\right]\left|\Psi\left(X_{N+1}, t^{\prime}\right)\right\rangle .
\end{gathered}
$$


The same can be done for Eq. (2a), replacing $\mathcal{K}\left(\mathbf{X}_{N+1}\right)$ and $\mathcal{D}\left(\mathbf{X}_{N+1}, t\right)$ by $\mathbf{h}(r)$ and $\mathbf{d}(r, t)$. It is always possible to add to $\mathcal{K}\left(\mathbf{X}_{N+1}\right)$ and subtract from $\mathcal{D}\left(\mathbf{X}_{N+1}, t\right)$ any time-independent operator without changing the form of Eq. (5). A customary and advantageous choice is to use the value of $\mathcal{D}\left(\mathbf{X}_{N+1}, t_{m}\right)$, where $t_{m}$ is the midpoint of the integration interval. By doing that, we may re-interpret the values of $\mathcal{K}\left(\mathbf{X}_{N+1}\right)$ and $\mathcal{D}\left(\mathbf{X}_{N+1}, t\right)$ to reflect that addition and subtraction.

The short iterative Lanczos (SIL) or Arnoldi method is directly employed in both the inner and outer region to evaluate the action of the matrix exponential on the vector known from the previous time step. Turning to the second term, for sufficiently short times only the part in the integral involving the Bloch operator contributes. This can be justified by noting that the term involving $\mathcal{D}\left(\mathbf{X}_{N+1}, t\right)$ would vanish if the integration was performed using the midpoint rule for sufficiently small time intervals.

To evaluate the term involving the Bloch operator, the suggested procedure is to expand $\mathcal{L}|\Psi(\mathbf{r}, t)\rangle$ in a power series and then evaluate the integral between $t_{0}$ and $t$ involving the exponential operator. It can be demonstrated that these terms may also be computed using a modification of the SIL suggested by Saad [36]. A further simplification is to use the external-region Hamiltonian rather than the internal-region Hamiltonian and the already computed outer-region vector to make that procedure even more computationally efficient. One thing to notice is that the integral involving the Bloch operator as well as the finite-difference operator contains information from points that are shared between the outer and inner regions. This requires some updating or iteration of that information at each time step.

For the remaining discussion, we assume that the short-time approximation is valid. There are separate investigations underway to remove this approximation, but those are beyond the scope of the present paper. However, it emerges from the above derivation that the accuracy of an $n$-step SIL or Arnoldi propagation is not proportional to the $n^{\text {th }}$ power of the step size for a time-dependent potential. The formal accuracy of the method is clearly second order, as follows from the midpoint formula.

Before concluding this section, some comments on the actual application of the two time-dependent R-matrix approaches is in order. The RMT grew out of a manydecades effort by Phil Burke and his collaborators to provide high-quality software for the solution of a broad class of problems in atomic and molecular physics. One should not underestimate the perseverance and dedication of all those scientists to develop the R-matrix software, which is now being used by researchers all over the world. In contrast, the BSR code was essentially due to a single individual, Oleg Zatsarinny. It was Oleg who maintained and added features to the code over a couple of decades. The Drake University / NIST effort to produce a code based on BSR to treat the interaction of EM radiation with atoms was neither planned for the long term nor was it initiated by Oleg himself. Nevertheless, Oleg was always helpful and did his best to enable the effort to move forward.

At the time, those of us interested in a TDBSR wanted to get out proof-of-principle results quickly and only later worry about questions of efficiency. The SIL had already been very successfully employed using a finite-element discrete-variable representation (FEDVR) approach in atomic He. While this approach could be generalized to more than two-electron systems, already having at our disposal the necessary ingredients from the BSR code enabled us to quickly study systems such as Ne and Ar. The idea was simple: take the field-free Hamiltonian and dipole matrices from the time-independent BSR calculations and propagate the initial bound state (also given by BSR as the initial vector) using the SIL. No attention was paid to an internal and external region. The R-matrix box was taken to be sufficiently large, so that after the SIL propagation we could already project onto asymptotic states. 


\section{Example Results}

Figure 1 shows one example of a comparison between the previous TDBSR [19] and new RMT calculations performed for the present paper. As expected, the agreement between the two sets of predictions is excellent, as is the agreement with the Floquet results of McKenna and van der Hart [37]. The latter, of course, are effectively a steadystate approach, i.e., the natural width of the window resonances and the effect of the intermediate $\left(3 \mathrm{p}^{5} 4 \mathrm{~s}\right)^{1} \mathrm{P}$ state, which acts as a stepping stone, are fully resolved. In the calculations with a finite-length pulse, on the other hand, the pulse width enters as well and smears out some of the sharp structures.

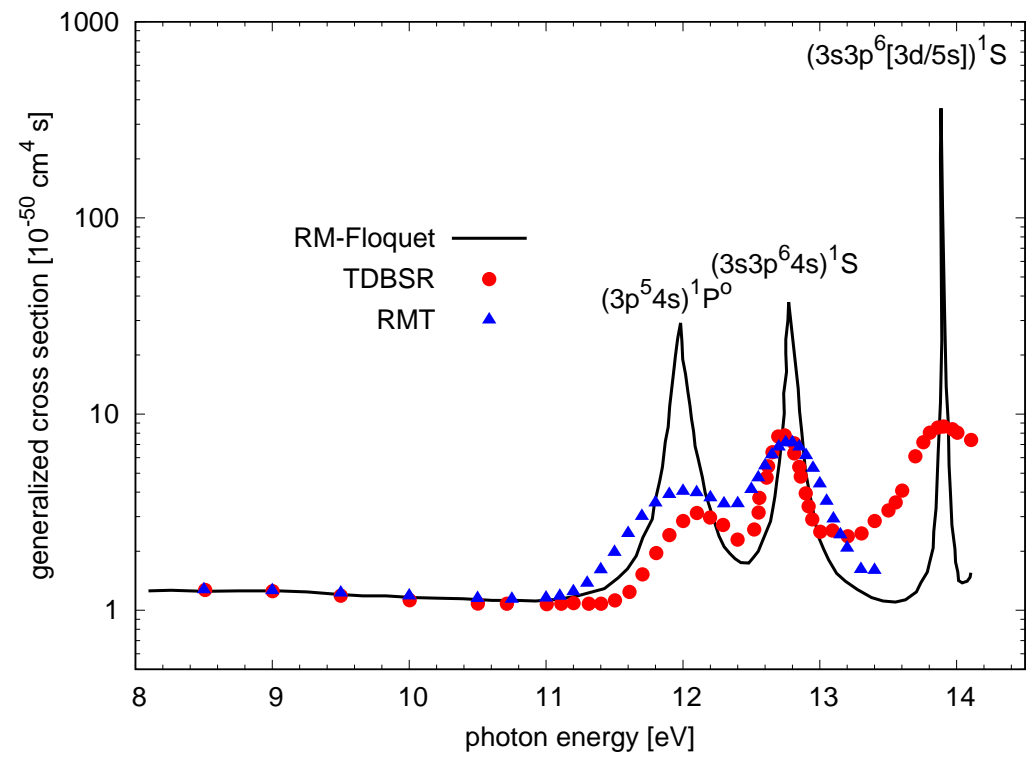

Figure 1. Generalized cross section for two-photon ionization of $\left.\operatorname{Ar}\left(3 p^{6}\right)^{1} S\right)$ as a function of photon energy. A 30-cycle laser pulse with a sine-squared field envelope and a peak intensity of $10^{12} \mathrm{~W} / \mathrm{cm}^{2}$ was used in the calculations. The Floquet results of McKenna and van der Hart [37] are compared with those from the previous TDBSR [19] and the current RMT calculations.

The remaining small disagreement between the TDBSR and the RMT results is due to the differences in the respective structure descriptions. Both the Floquet and the RMT results were obtained with the structure description developed by Burke and Taylor [38], who designed pseudo-orbitals to account for the strong term dependence of the $3 s$ and $3 p$ orbitals in the neutral $\left(3 s^{2} 3 p^{6}\right)^{1} S$ ground state as well the coupled final ionic $\left(3 s^{2} 3 p^{5}\right)^{2} \mathrm{P}$ and $\left(3 s 3 p^{6}\right)^{2} S$ states. This was particularly important to obtain accurate ionization potentials as well as the position (i.e., the excitation energy) of the intermediate $\left(3 p^{5} 4 s\right)^{1} \mathrm{P}$ state. No such special effort was devoted to this issue in the TDBSR calculations, but apparently a sufficient number of short-range orbitals were included in this case to obtain accurate results. This is definitely a topic that BSR, in principle, can handle extremely well. On the other hand, if only the bare minimum of orbitals is included, problems can arise. An example is depicted in Fig. 4 of Guan et al. [19] in a similar calculation for two-photon ionization of $\mathrm{Ne}\left(2 \mathrm{p}^{6}\right)^{1} \mathrm{~S}$. In that very first TDBSR calculation, the ionization potential is too small, i.e., the ground state lies too high, and hence the theoretical excitation energy of the $\left(2 \mathrm{p}^{5} 3 \mathrm{~s}\right)^{1} \mathrm{P}$ state is too small.

\section{Summary and Outlook}

In this contribution, we revisited some of the early work on the extension of the R-matrix (close-coupling) method, which was originally designed to treat - accurately and efficiently - electron and photon collisions with atoms, ions, and molecules in a steady-state scenario, to include the reaction of these targets to short-pulse intense electromagnetic radiation in a time-dependent framework. While the RMT code of 


\section{Abbreviations}

222 TDSE time-Dependent Schrödinger Equation BSR B-Spline R-Matrix

RMT R-Matrix with Time Dependence

223 TDBSR Time-Dependent B-Spline R-matrix

SIL Short Iterative Lanczos

XUV Extreme Ultraviolet

\section{References}

1. Lane, A.M.; Robson, D. Comprehensive Formalism for Nuclear Reaction Problems. I. Derivation of Existing Reaction Theories. Phys. Rev. 1966, 151, 774-787. doi:10.1103/PhysRev.151.774.

2. Lane, A.M. The application of Wigner's R-matrix theory to atomic physics. J. Phys. B. At. Mol. Phys. 1986, 19, 253-257. doi:10.1088/0022-3700/19/2/014.

3. Burke, P.G.; Hibbert, A.; Robb, W.D. Electron scattering by complex atoms. J. Phys. B. At. Mol. Phys. 1971, 4, 153-161. doi:10.1088/0022-3700/4/2/002.

4. Berrington, K.A.; Burke, P.G.; Robb, W.D. The scattering of electrons by atomic nitrogen. J. Phys. B. At. Mol. Phys. 1975, 8, 2500-2511. doi:10.1088/0022-3700/8/15/012.

5. Burke, P.G.; Robb, W.D. The R-Matrix Theory of Atomic Processes. Adv. At. Molec. Phys. 1976, 11, 143-214. doi:10.1016/S00652199(08)60030-5.

6. Burke, P.G. R-Matrix Theory of Atomic Collisions; Springer Series on Atomic, Optical, and Plasma Physics 61, Springer-Verlag, 2011. doi:10.1007/978-3-642-15931-2.

7. Schneider, B. R-matrix theory for electron-atom and electron-molecule collisions using analytic basis set expansions. Chem. Phys. Lett. 1975, 31, 237-241.

8. Schneider, B.I. R-matrix theory for electron-molecule collisions using analytic basis set expansions. II. Electron- $\mathrm{H}_{2}$ scattering in the static-exchange model. Phys. Rev. A 1975, 11, 1957.

9. Burke, P.G.; Mackey, I.; Shimamura, I. R-matrix theory of electron-molecule scattering. Journal of Physics B: Atomic and Molecular Physics 1977, 10, 2497-2512. doi:10.1088/0022-3700/10/12/027.

10. Mašín, Z.; Benda, J.; Gorfinkiel, J.D.; Harvey, A.G.; Tennyson, J. UKRmol+: A suite for modelling electronic processes in molecules interacting with electrons, positrons and photons using the R-matrix method. Comp. Phys. Commun. 2020, $249,107092$. doi:https://doi.org/10.1016/j.cpc.2019.107092. 
11. Zatsarinny, O. BSR: B-spline atomic R-matrix codes. Comp. Phys. Commun. 2006, 174, 273-356. doi:10.1016/j.cpc.2005.10.006.

12. Zatsarinny, O; Bartschat, K. The B-spline R-matrix method for atomic processes: application to atomic structure, electron collisions and photoionization. J. Phys. B. At. Mol. Opt. Phys. 2013, 46, 112001. doi:10.1088/0953-4075/46/11/112001.

13. Froese Fischer, C.; Idrees, M. Spline algorithms for continuum functions. Comp. in Phys. 1989, 3, 53-58. doi:10.1063/1.168325.

14. Berrington, K.A.; Eissner, W.; Norrington, P.H. RMATRIX-I: Belfast Atomic R-Matrix Codes. Comp. Phys. Commun. 1995, 92, 290. doi:https:/ /doi.org/10.1016/0010-4655(95)00123-8.

15. Wragg, J.; Ballance, C.; van der Hart, H. Breit-Pauli R-Matrix approach for the time-dependent investigation of ultrafast processes. Comp. Phys. Commun. 2020, 254, 107274. doi:https://doi.org/10.1016/j.cpc.2020.107274.

16. Bray, I.; Fursa, D.; Kadyrov, A.; Stelbovics, A.; Kheifets, A.; Mukhamedzhanov, A. Electron- and photon-impact atomic ionisation. Phys. Rep. 2012, 520, 135-174. Electron- and photon-impact atomic ionisation, doi:https://doi.org/10.1016/j.physrep.2012.07.002.

17. Bartschat, K.; Hudson, E.T.; Scott, M.P.; Burke, P.G.; Burke, V.M. Electron - atom scattering at low and intermediate energies using a pseudo-state/ R-matrix basis. J. Phys. B: At. Mol. Phys. 1996, 29, 115.

18. Ren, X.; Pflüger, T.; Ullrich, J.; Zatsarinny, O.; Bartschat, K.; Madison, D.H.; Dorn, A. Erratum: Low-energy electron-impact ionization of argon: Three-dimensional cross section [Phys. Rev. A 85, 032702 (2012)]. Phys. Rev. A 2015, 92, 019901. doi:10.1103/PhysRevA.92.019901.

19. Guan, X.; Zatsarinny, O.; Bartschat, K.; Schneider, B.I.; Feist, J.; Noble, C.J. General approach to few-cycle intense laser interactions with complex atoms. Phys. Rev. A 2007, 76, 053411. doi:10.1103/PhysRevA.76.053411.

20. Guan, X.; Zatsarinny, O.; Noble, C.J.; Bartschat, K.; Schneider, B.I. Time-dependent B-spline R-matrix approach to double ionization of atoms by XUV laser pulses. J. Phys. Conf. Ser. 2009, 194, 032027. doi:10.1088/1742-6596/194/3/032027.

21. Guan, X.; Bartschat, K.; Schneider, B.I. Dynamics of two-photon double ionization of helium in short intense xuv laser pulses. Phys. Rev. A 2008, 77, 043421. doi:10.1103/PhysRevA.77.043421.

22. Feist, J.; Nagele, S.; Pazourek, R.; Persson, E.; Schneider, B.I.; Collins, L.A.; Burgdörfer, J. Nonsequential two-photon double ionization of helium. Phys. Rev. A 2008, 77, 043420. doi:10.1103/PhysRevA.77.043420.

23. Feist, J.; Zatsarinny, O.; Nagele, S.; Pazourek, R.; Burgdörfer, J.; Guan, X.; Bartschat, K.; Schneider, B.I. Time delays for attosecond streaking in photoionization of neon. Phys. Rev. A 2014, 89, 033417. doi:10.1103/PhysRevA.89.033417.

24. Burke, P.G.; Burke, V.M. Time-dependent R-matrix theory of multiphoton processes. J. Phys. B. At. Mol. Opt. Phys. 1997, 30, L383-L391. doi:10.1088/0953-4075/30/11/002.

25. Nikolopoulos, L.A.A.; Parker, J.S.; Taylor, K.T. Combined R-matrix eigenstate basis set and finite-difference propagation method for the time-dependent Schrödinger equation: The one-electron case. Phys. Rev. A 2008, 78, 063420. doi:10.1103/PhysRevA.78.063420.

26. Lysaght, M.A.; van der Hart, H.W.; Burke, P.G. Time-dependent R-matrix theory for ultrafast atomic processes. Phys. Rev. A 2009, 79, 053411. doi:10.1103/PhysRevA.79.053411.

27. Parker, J.S.; Doherty, B.J.S.; Taylor, K.T.; Schultz, K.D.; Blaga, C.I.; DiMauro, L.F. High-Energy Cutoff in the Spectrum of Strong-Field Nonsequential Double Ionization. Phys. Rev. Lett. 2006, 96, 133001. doi:10.1103/PhysRevLett.96.133001.

28. Moore, L.; Lysaght, M.; Nikolopoulos, L.; Parker, J.; van der Hart, H.; Taylor, K. The RMT method for many-electron atomic systems in intense short-pulse laser light. J. Mod. Opt. 2011, 58, 1132-1140. doi:10.1080/09500340.2011.559315.

29. Clarke, D.D.A.; Armstrong, G.S.J.; Brown, A.C.; van der Hart, H.W. $<$ math $><$ mi $>$ R $</$ mi $></$ math $>-$ matrix-with-timedependence theory for ultrafast atomic processes in arbitrary light fields. Phys. Rev. A 2018, 98, 053442. doi:10.1103/PhysRevA.98.053442.

30. Benda, J.; Gorfinkiel, J.D.; Mašín, Z.; Armstrong, G.S.J.; Brown, A.C.; Clarke, D.D.A.; van der Hart, H.W.; Wragg, J. Perturbative and nonperturbative photoionization of $\mathrm{H}_{2}$ and $\mathrm{H}_{2} \mathrm{O}$ using the molecular R-matrix-with-time method. Phys. Rev. A 2020, 102, 052826. doi:10.1103/PhysRevA.102.052826.

31. Wragg, J.; Clarke, D.D.A.; Armstrong, G.S.J.; Brown, A.C.; Ballance, C.P.; van der Hart, H.W. Resolving Ultrafast Spin-Orbit Dynamics in Heavy Many-Electron Atoms. Phys. Rev. Lett. 2019, 123, 163001. doi:10.1103/PhysRevLett.123.163001.

32. Brown, A.C.; Armstrong, G.S.; Benda, J.; Clarke, D.D.; Wragg, J.; Hamilton, K.R.; Mašín, Z.; Gorfinkiel, J.D.; van der Hart, H.W. RMT: R-matrix with time-dependence. Solving the semi-relativistic, time-dependent Schrödinger equation for general, multielectron atoms and molecules in intense, ultrashort, arbitrarily polarized laser pulses. Comp. Phys. Commun. 2020, 250, 107062. doi:https://doi.org/10.1016/j.cpc.2019.107062.

33. Github repository for the B-Spline atomic R-Matrix (BSR) code. https://github.com/zatsaroi/BSR3; Accessed Jan. $24,2022$.

34. Gitlab repository for the R-Matrix with Time-dependence (RMT) code. https://gitlab.com/Uk-amor/RMT/rmt; Accessed Jan. 24, 2022.

35. AMOS Gateway: A Portal for Research and Education in Atomic, Molecular, and Optical Science. https://amosgateway.org/; accessed Jan. 20, 2022.

36. Saad, Y. Analysis of Some Krylov Subspace Approximations to the Matrix Exponential Operator. SIAM Journal on Numerical Analysis 1992, 29, 209-228.

37. McKenna, C.; van der Hart, H.W. Multiphoton ionization cross sections of neon and argon. J. Phys. B. At. Mol. Opt. Phys. 2003, 37, 457-470. doi:10.1088/0953-4075/37/2/013.

38. Burke, P.G.; Taylor, K.T. R-matrix theory of photoionization. Application to neon and argon. J. Phys. B. At. Mol. Opt. Phys. 1975, 8, 2620 . 
39. Bartschat, K.; Fischer, C.F.; Grum-Grzhimailo, A.N. Oleg Zatsarinny (1953-2021): Memories by His Colleagues. Atoms 2021, 9. doi:10.3390/atoms9040109. 